\title{
Don't Go Viral: An Evidence-Based Practice Project for Increasing Seasonal Influenza Vaccine Rates on a College Campus
}

\author{
Jennifer J. Wasco, DNP, RN \\ Chatham University
}

\begin{abstract}
Aim: To describe the effectiveness of an evidence-based practice (EBP) project on a college campus using an educational campaign that leveraged technology as well as traditional marketing strategies for the dissemination of valuable, reliable health information about the seasonal influenza virus and seasonal influenza vaccination.

Background: Despite the typical seasonal influenza vaccine effectiveness (VE) of approximately $50 \%$ - 60\% (Centers for Disease Contol and Prevention, 2016c), some groups of individuals, such as young adults, do not take advantage of obtaining the immunization for a variety of reasons.

Methods: Students $(\mathrm{N}=55)$ at a small private university in southwestern Pennsylvania agreed to participate in an EBP project between September 2015 and October 2015. Project participants were sent a link to an animated online video about seasonal influenza, and an optional text message reminder was sent to encourage vaccination against the virus. An electronic survey was utilized to identify demographics and confirm the effectiveness of the campaign methods.

Results: Due to the limited number of survey responses, a broad scale conclusion of intervention effectiveness cannot be applied against the larger college student population nationally. However, the results from the participants who viewed the online educational video showed a favorable reaction to this type of intervention.

Conclusions: Based on the EBP project results, when developing health-related material and programming, consideration needs to be given to the college student population's preferences on learning. Given the computer usage statistics of the college-age population and the results of this project, the matching of the video to the population's preferred medium is supported.
\end{abstract}

Submitted 4 December 2016; accepted 2 March 2017.

Keywords: college students, seasonal influenza, evidence-based practice, health decision aids, health engagement

Seasonal influenza, or most commonly known as the flu, is a contagious respiratory illness caused by viruses infecting the nose, throat, and lungs. The virus results in a mild to severe illness, and at times can lead to death (Centers for Disease Control and Prevention [CDC], 2016a). In the United States, 5\% - 20\% of the population contracts seasonal influenza each year (Molinari et al., 2007). The CDC's (2016b) position is the seasonal influenza vaccine is the best way to prevent the virus. Obtaining the seasonal influenza vaccination reduces doctor's visits for seasonal influenza-related illnesses by appropriately 50\% - 60\% among the overall population when the viruses are spreading in the community (CDC, 2016d).

Despite vaccine effectiveness (VE) and recommendations for receiving the annual vaccination, some individuals continue to hold misconceptions, have a lack of knowledge or motivation about obtaining the vaccination (Benjamin \& Bahr, 2016). According to Bednarczyk et al. (2014) and the National Foundation for Infectious Diseases (NFID; 2016), young adults, including college students, are one group that holds these misunderstandings about the benefits of vaccination. An American College Health Association (ACHA) executive summary published in 2013, identified only $43.1 \%$ of students nationally reported receiving the seasonal influenza vaccine in the 12 months prior. In a 2011 study, 32.5\% of college students in the United States between the ages of 18 - 24 felt they were not at risk for seasonal influenza because they were young and healthy (Ramsey \& Marczinski, 2011). Additionally, a field experiment conducted by Bronchetti, Huffman, and Magenheim (2015) indicated that a lack of follow through on intentions to be vaccinated is a major reason for the low seasonal influenza vaccination uptake in the college population. Finally, 
according to Cheney and John (2013), the health beliefs of the individuals play a vital role in accepting or resisting the seasonal influenza vaccine.

In a 2011 report by Pew Research Center, 96\% of undergraduate students and $99 \%$ of graduate students own a cell phone. Similarly, laptop ownership for undergraduate students was $88 \%$ and $73 \%$ for graduates. As Bronchetti et al. (2015) identified, students were twice as likely to take action utilizing information obtained through information technology such as video email messaging as compared to traditional methods of health information provided through pamphlets and handouts. The mismatch between the SHS offices' methods of information delivery and the students' access to technology was identified as a potential barrier to health promotion and preventative health care actions of students on campus.

The problem of low vaccination rates among college students is compounded due to the rapid spread of seasonal influenza on college campuses due to the highly populated living accommodations and classroom environments (Yang, 2012). According to Nichol et al. (2016), college students can experience seasonal influenza for eight days or more resulting in the loss of academic productivity (Mullins et al., 2011). As many as 13.2\% of students reported seasonal influenza illnesses affected their academic performance (ACHA, 2013). Contracting seasonal influenza also puts limitations on the interpersonal socialization opportunities of the student population (Walgreens, 2013). Additionally, there are increased rates of healthcare utilization in the college population (National Foundation for Infectious Diseases [NFID], 2016) comprising the overall estimated $\$ 10.4$ billion a year in direct medical costs (Molinari et al., 2007).

\section{PURPOSE OF PROJECT}

On the university campus, the Student Health Services (SHS) office provides traditional access to information about seasonal influenza. The information is disseminated through flyers, brochures and face-to-face counseling by office support staff. The campus' SHS office does not currently provide technology-based health educational materials to the student population.

The purpose of this project was to assess the preliminary effectiveness of a campus technology-based healthcare educational program about seasonal influenza and the seasonal influenza vaccine. This EBP project utilized traditional educational methods using flyers supplemented with email, video and text messaging focusing on improving a student's knowledge about the seasonal influenza virus and vaccination. The focus on electronic means to disseminate the educational content was supported by the statistics regarding the ownership rates of electronic devices within the population.

\section{METHODS}

\section{Development of Educational Materials}

The development of the educational materials and dissemination of materials was based on the theoretical framework of the health belief model (HBM; Rosenstock, 1974) and the EBP theory, diffusion of innovation (Rogers, 1960). First, understanding the HBM theory provides necessary insights into why people take health actions, which in turn allows for an improved development of educational content. In a study by Cheney and John (2013), individuals that had the perceived threat of contracting the virus were 5.4\% more likely to get vaccinated. Even though this particular study focused on identifiable high-risk groups, ethnic minority, low socioeconomic status and chronically ill adults, the use of this information was applied to this project's educational materials as the college population is considered high risk due to close living conditions, crowded social situations, and highly populated classrooms.

In similar fashion, the diffusion of innovation theory discussed the manner in which new information spread among a population and was the basis for the educational material delivery to the population. There are five distinct steps: awareness, persuasion, decision, implementation, and confirmation (Figure 1). Educational materials created correlated with each step within the diffusion of innovations theory. Bulletin board campaigns addressed the initial step of increasing awareness. In the second step, individuals were encouraged to form an opinion regarding 
the seasonal influenza vaccination after receiving a web-based animated educational video with accurate facts and information. The individual was also invited to receive an optional, one-time text message reminding them to get the vaccination during enrollment which falls under the decision-making process because it provided the dates and times when the SHS office was open. The fourth step is where the student takes the preventative actions, and the final step of the process is where the population finds confirmation in their actions or inactions, and participant feedback was obtained via the post-participant questionnaire.

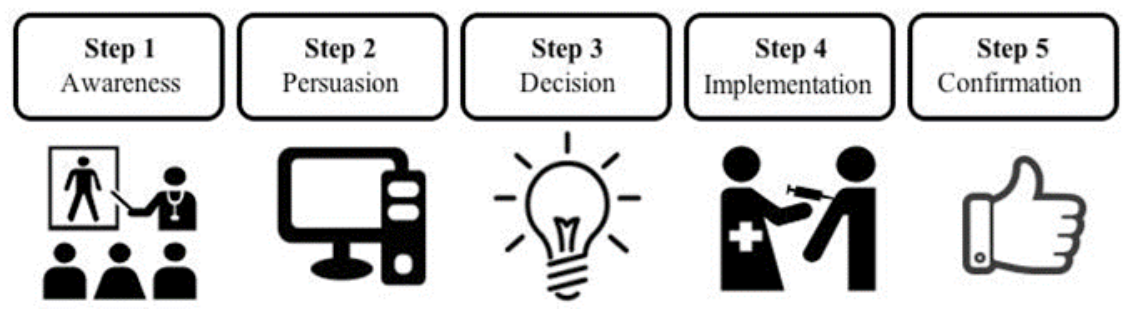

Figure 1. Decision-making process using the Diffusion of Innovations Theory.

\section{Practice Setting and Participants}

The practice setting for the EBP project was a small private co-educational university in southwestern Pennsylvania. In October 2015, the total student enrollment was 2,224 (undergraduates: 1,034 and graduate students: 1,190). There was an increase in the number of undergraduate students (+104) and a decrease of graduate students (-14) comparing October of 2014. The increase in undergraduate students increased the potential for more exposure to illness during the academic year.

Participation in the project was voluntary. Recruited participants included both undergraduate and graduate students 18 years of age and older. There was no restriction to gender, race, religion, or nationality. Students enrolled in the physician assistant (PA) program were not included in the project as the seasonal influenza vaccination is a program requirement. Also, the employees of the University were not included because the employee health insurance plans offer incentives for participating in preventative health measures. A total of 55 individuals agreed to participate in the EBP change project. Of the 55 participants, 14 individuals $(25 \%)$ completed the project survey.

\section{Implementation Description}

Potential participants were made aware of the EBP change project through flyers located on campus bulletin boards. The flyers rotated on a pre-planned bi-weekly basis early in the semester and then changed to a weekly schedule the middle of the semester. (See Figure 2 for examples of posters increasing awareness).
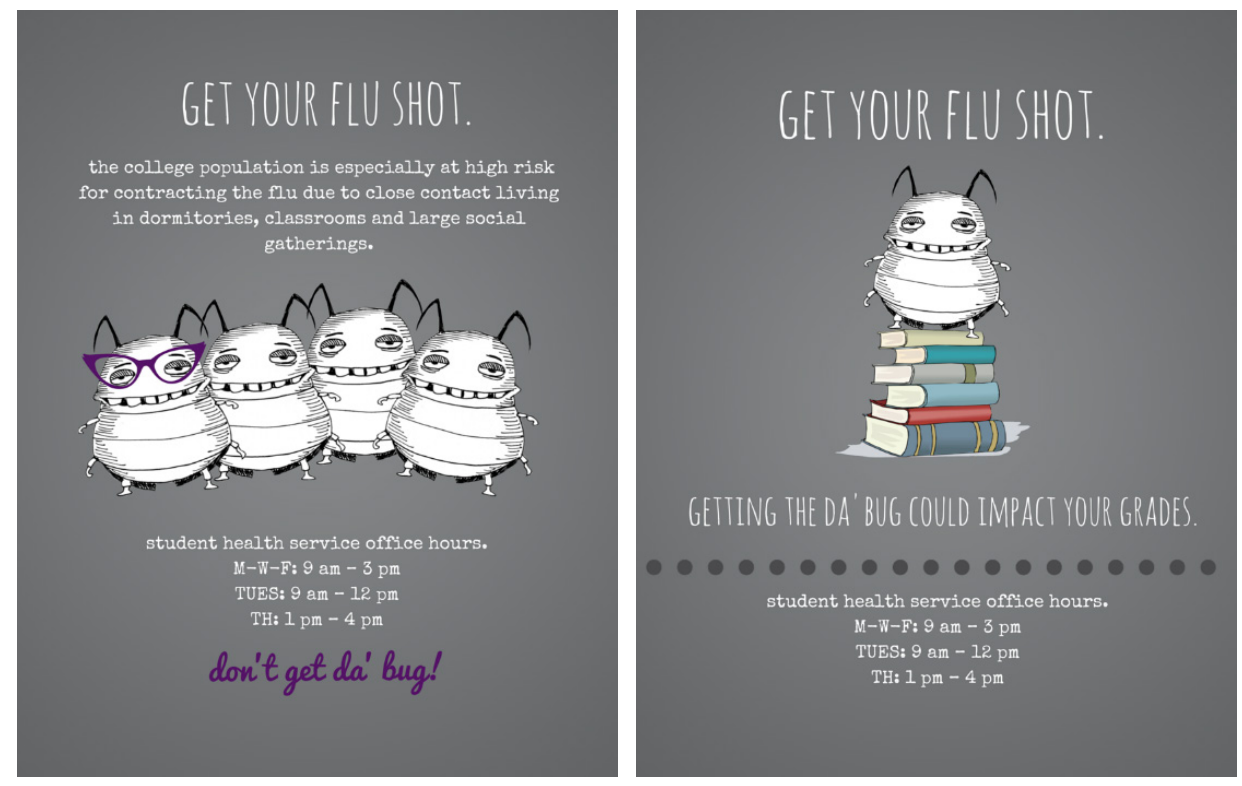

Figure 2. Example imaging and messaging of bulletin board flyers. 
An information table was set up at campus events sponsored by the Office of Student Affairs including a welcome dinner, a mental health symposium, a midnight madness athletic event and during peak cafeteria hours on campus properties. Once an interested individual presented themselves at the information table, an invitation to participate in the project was provided. If the person indicated an interest in participating in the project, the informed consent form that was approved by the university's Intuitional Review Board (IRB) was provided. All questions about the project and informed consent were answered at the time of enrollment. Also collected at that time was the consenting individual's email address and optional mobile phone number. The collection of this type of information was necessary for the individual to participate in the interventions related to this project. All individuals who visited the information regardless of project enrollment were provided with a postcard-sized handout that listed the SHS's office hours.

The EBP change project was carried out through three phases. During the first phase, the participants were emailed a link to the animated educational video within seven days of an agreement to participate by the project manager (PM). The animated online video was four minutes long and addressed information about the seasonal influenza virus and the seasonal influenza vaccination (Figure 3). The "Don't Go Viral" video was accessed by utilizing a link to the posted video that was embedded in the email messaging.

\section{Animated Educational Video Content Outline}

I. Introduction
a. Welcome

II. Facts about the flu
a. What is the flu?
b. Impact of the flu
c. College students at high risk for the flu; close living conditions

III. Flu prevention
a. Hand Washing: Everyday prevention activity
b. Vaccination: Best prevention step

IV. Debunking myths
a. The vaccine causes flu illness
b. Young and healthy people do not need the vaccine
c. Misunderstanding about the last year's vaccine effectiveness

V. Barriers to vaccination
a. Convenience
b. Cost
c. Misunderstanding about the vaccine's effectiveness last season

VI. Talking with your health care provider
a. Contact your healthcare provider for more information about the flu
b. Possible health reasons not to get vaccinated

VII. Flu season starts soon

a. Best month to get vaccinated

Figure 3. Content outline for the educational video.

In the second phase of the project, a text message reminder using TellMyCell ${ }^{\circledR}$ Short Message Service (SMS) software was sent to the mobile phone number provided by the project participant. The text message was sent to the project participant based on their date of enrollment in the project. The text message reminder read "This is your reminder to get your seasonal influenza shot! October is the best month to be protected against the seasonal influenza virus." 
Finally, phase three of the project included an email to project participants with an embedded link to a 16-question Qualtrics survey created by the PM (Figure 4). The survey obtained project participant demographics and measured the effectiveness of the marketing campaign by assessing the project participant's intent to be protected from the seasonal influenza virus by receiving the seasonal influenza vaccine during the 2015 - 2016 influenza season.

\section{Flu Vaccination Survey Questions}

1. What is your gender?

2. What is your age range?

3. What type of degree are you seeking?

4. What is your housing status?

5. Did you get the flu shot last year?

6. Do you remember seeing the flu posters on campus this fall?

7. If you remember seeing the flu posters on campus, how useful were the flu posters in influencing your decision to get the flu shot this season?

8. Did you view the animated educational video about the flu?

9. If you viewed the animated educational video, how useful was it about the flu in providing you with a better understanding about the flu virus and the flu shot?

10. Did you receive the text message reminder to get the flu shot?

11. If you received the text message, how useful was it in reminding you to get the flu shot?

12. Did you get the flu shot yet this year?

13. If you got the flu shot already this year, where did you get it?

14. If you did not get the flu shot yet this year, are you planning to get it?

15. If you will get the flu shot, where are you planning to do it?

Figure 4. Participant Survey.

\section{RESULTS}

The project's data was collected using the Qualtrics survey software, and the results were downloaded using Microsoft Excel $^{\mathbb{R}}$ spreadsheets. Calculations were done using Microsoft Excel formulas to arrive at the values for the project. The summary of that data was entered into Microsoft Excel spreadsheet for analysis.

A total of 55 individuals agreed to participate in the EBP change project. Of the 55 participants, fourteen individuals $(25 \%)$ completed the survey. All of the initial project participants provided an email address to receive the animated video and survey. In addition, 37 of the 55 participants provided a mobile phone number to receive the text message reminder upon enrollment. The survey respondents consisted of three 
male students and eleven female students. The participants ranged in age from 18 to 29 years with 65\% of the students being 21 years old or younger. There were seven undergraduate students and seven graduate students (see Table 1).

Table 1

Summary of Project Participant Demographics

\begin{tabular}{lll}
\hline Gender & $n=$ & $\%$ \\
\hline Male & 3 & $21.43 \%$ \\
Female & 11 & $78.57 \%$ \\
\hline Age Range & & \\
$\quad 18-19$ & 5 & $35.71 \%$ \\
$20-21$ & 4 & $28.57 \%$ \\
$22-24$ & 3 & $21.43 \%$ \\
$\quad 25-29$ & 2 & $14.29 \%$ \\
Degree Status & & \\
$\quad$ Undergraduate & 7 & $50 \%$ \\
$\quad$ Graduate & 7 & $50 \%$ \\
\hline Housing Status & & \\
On Campus & 10 & $71.43 \%$ \\
Off Campus or Commuting & 4 & $28.57 \%$ \\
\hline School of Enrollment & & \\
Arts, Science, \& Business & 6 & $42.86 \%$ \\
Health Sciences & 6 & $42.86 \%$ \\
Sustainability & 2 & $14.29 \%$
\end{tabular}

The EBP project results focused on the impact of the educational messages and how they were received and valued by the project participants. The survey questions asked the participant to provide insight into the usefulness of the campaign materials using a Likert scale. The mean of the Likert scale responses was determined, and the following means were compared to determine if different components of the intervention were more influential in changing the participant's perception regarding the seasonal influenza vaccination (see Figure 5).

\section{Bulletin Board Flyers}

The first question about educational materials asked participants if they remembered seeing the flu posters on campus bulletin boards. Thirteen of the participants (93\%) indicated that they remembered seeing the posters. The mean rating on the usefulness of the posters on influencing personal health decision making to receive the flu vaccination was 6.83. The result that thirteen out of the fourteen participants were aware of the flyers indicates that this phase of the marketing campaign was successful at informing the student population of the upcoming flu season.

\section{Animated Educational Video}

The second component of the project was the dissemination of the animated video. The animation was seen by 9 of the 14 project participants. This aspect of the project had a greater influence upon the population, scoring an 8.1 on the 10-point Likert scale. The score suggests that the video was more influential among the population, which is consistent with the population's lesser scores applied to the printed materials. The score may also be more indicative of the population's reliance on computers for such messaging. 


\section{Text Message Reminder}

The final questions asked participants about the effectiveness of the text message reminder. Five of the participants $(38 \%)$ indicated that they received the text message reminder. The mean score regarding the usefulness of the text message in reminding them to obtain the flu vaccination was 6.4. The average score of the respondents on this question was $6.4(n=5)$. The fact that this had the lowest participation rate and consisted only of a single text message may help explain why the text message was scored about as effective as the flyers.

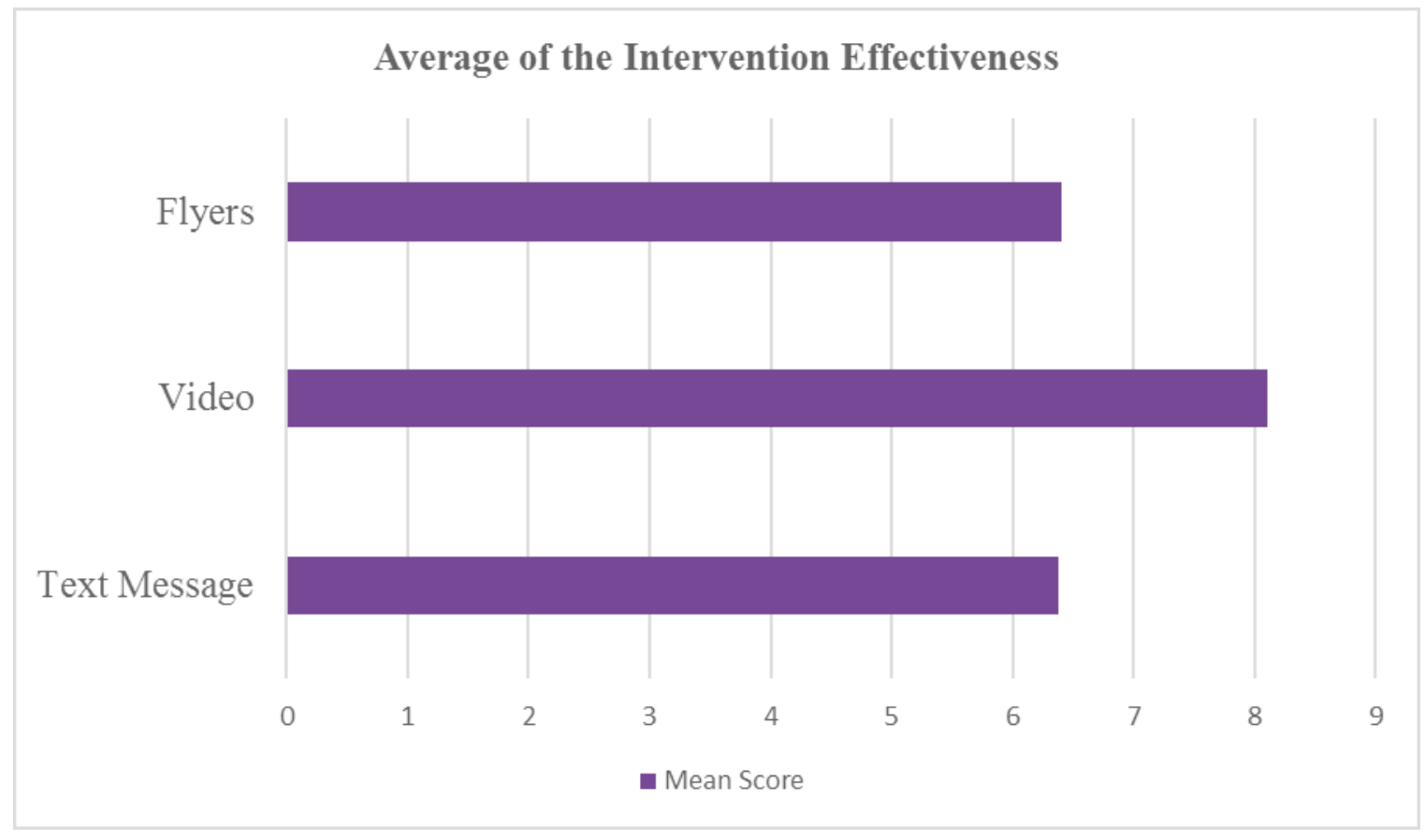

Figure 5. Average of the intervention effectiveness.

Due to the limited number of survey responses, a broad scale conclusion of intervention effectiveness cannot be applied against the larger college student population nationally. However, the results from the participants who viewed the online educational video showed a favorable reaction to this type of intervention. Given the computer usage statistics of the college-age population (Pew Internet, 2011) and the results of this project, the matching of the video to the population's preferred medium is supported.

\section{COMMENT}

Understanding a population's preferences for learning is essential for the deployment of an intervention as seen by the results of this EBP project. The flyers were posted in high-traffic areas may have been sufficient to increase awareness of the project to the student population. However, the flyers were not deemed to be highly influential especially when compared to the outcome of the online animated video score. The discrepancy in scores between the traditional paper media (flyers) and video animation methods underscores the importance of tailoring educational messages to the population using methods the population prefers. While understanding the success of the various educational methods was the purpose of this project, the underlying health aim was to increase the population's health through increased vaccination rates. The survey results indicated that only three of the participants obtained the flu vaccination in the prior year, and that six had obtained the vaccination in the current year with two more intending to obtain the vaccination. The increase can be viewed favorably in light of the high scoring of the educational content. Although no direct conclusion can be drawn that the individuals positively decided to obtain a vaccination directly because of the educational campaign, the increase does suggest that tailoring the educational content to the population using the preferred media and delivery method is advisable. 


\section{FUTURE PROJECTS}

Future projects include the development of additional topics for the promotion of health and wellness with the goal of delivering the content electronically to the student body. An electronic library of resources could be made available through the SHS website. The methods that were used in this EBP change project could be replicated on other topics to obtain data on what the students are viewing. The SHS office could provide service changes based in part on what resources were being used via the electronic methods.

\section{CONCLUSIONS}

The development of health-related material and programming should be tailored to the learning preferences of the community. This EBP project's outcomes identified that by leveraging the technology most valued and utilized to acquire knowledge among a college population. Therefore, a university can strategically plan to disseminate health information efficiently in this manner to promote a healthy community. 


\section{REFERENCES}

American College Health Association (ACHA; 2013). American College Health Association-National College Health Assessment II: Fall 2013 Reference Group Executive Summary. Linthicum, MD: American College Health Association. Retrieved from http:/ / www.acha-ncha.org/docs/ACHA-NCHA-II_ReferenceGroup_ExecutiveSummary_Fall2013.pdf

Bednarczyk, R., Sickler, H., Chu, S., Shaw, J., Nadeau, J., \& McNutt, L.A. (2014, October). Low uptake of seasonal influenza vaccine among university students: Evaluating predictors beyond cost and safety concerns [Abstract]. Poster session presented at the annual scientific meeting IDWeek, Philadelphia, PA. Abstract retrieved from https://idsa.confex. com/idsa/2014/webprogram/Paper47003.html\#.

Benjamin, S.M., \& Bahr, K.O., (2016). Barriers Associated with Seasonal Influenza Vaccination among College Students. Seasonal Influenza Research and Treatment, 2016. 4248071. https://doi.org/10.1155/2016/4248071

Bronchetti, E. T., Huffman, D. B., \& Magenheim, E. (2015) Attention, intentions, and follow-through in preventive health behavior: Field experimental evidence on seasonal influenza vaccination. Journal of Economic Bebavior \& Organization, 11, 6270-6291. https://doi.org/10.1016/j.jebo.2015.04.003

Centers for Disease Control and Prevention (CDC; 2016a). Flu Symptoms and Complications. Retrieved from https://www.cdc.gov/flu/about/disease/complications.htm

Centers for Disease Control and Prevention (CDC; 2016b). Key Facts about Seasonal Influenza Vaccine. Retrieved from https://www.cdc.gov/flu/protect/keyfacts.htm

Centers for Disease Control and Prevention (CDC; 2016c). Vaccine effectiveness: How well does the seasonal influenza vaccine work? Retrieved from http://www.cdc.gov/seaonal influenza/about/qa/vaccineeffect.htm

Centers for Disease Control and Prevention (CDC; 2016d). Vaccination Benefit Publications. Retrieved from https://www.cdc.gov/flu/pdf/freeresources/general/flu-vaccine-benefits-update.pdf

Cheney, M.K., \& John, R. (2013). Underutilization of seasonal influenza vaccine: A test of the health belief model. SAGE Open, 3(2). https://doi.org/10.1177/2158244013484732

Molinari, N. M., Ortega-Sanchez, I. R., Messonnier, M. L., Thompson, W. W., Wortley, P. M., Weintraub, E., \& Bridges, C. B. (2007). The annual impact of seasonal influenza in the US: Measuring disease burden and costs. Vaccine, 25(27), 50865096. https://doi.org/10.1016/j.vaccine.2007.03.046

Mullins, J., Cook, R., Rinaldo, C., Yablonsky, E., Hess, R., \& Piazza, P. (2011). Seasonal influenza-like illness among university students: Symptom severity and duration due to seasonal influenza virus infection compared to other etiologies. Journal of American College Health, 59(4), 246-251. https://doi.org/10.1080/07448481.2010.502197

National Foundation for Infectious Diseases Report (2016). Addressing the Challenges of Seasonal Influenza Vaccination on US College Campuses. Retrieved from http://www.nfid.org/

Nichol, K.L., Tummers, K., Hoyer-Leitzel, A., Marsh, J., Moynihan, M., \& McKelvey, S. (2010). Modeling Seasonal Seasonal Influenza Outbreak in a Closed College Campus: Impact of Pre-Season Vaccination, In-Season Vaccination and Holidays/Breaks. PLoS ONE 5(3): e9548. https://doi.org/10.1371/journal.pone.0009548

Pender, N.J., Murdaugh, C.L., \& Parsons, M.A. (2015). Health Promotion in Nursing Practice (7th ed.). Boston, MA: Person

Pew Internet (2011). College students and technology. Retrieved from http://www.pewinternet.org/2011/07/19/collegestudependernts-and-technology/

Ramsey, M., \& Marczinski, C. (2011). College students' perceptions of H1N1 seasonal influenza risk and attitudes towards vaccination. Vaccine, 29(44), 7599-7601. https://doi.org/10.1016/j.vaccine.2011.07.130

Rogers, E. M. (1960). Diffusion of innovations. New York, NY: Free Press.

Rosenstock, I.M. (1974). Historical origins of the health belief model. Health Education \& Behavior, 2(4), 328-335. https://doi.org/10.1177/109019817400200403 
Walgreens (2013). Walgreens 2013 seasonal infuenza impact report. Retrieved from http://www.multivu.com/players/ English/62923-walgreens-flu-season2013/

Yang, Z.J. (2012). Too scared or too capable? Why do college students stay away from the H1N1 vaccine? Risk Analysis, 32(10), 1703-1716. https://doi.org/10.1111/j.1539-6924.2012.01799.x

Author correspondence may be addressed to:

Jennifer J. Wasco, DNP, RN

Practice Experience Coordinator, Nursing Programs, School of Health Sciences

Chatham University, Pittsburgh, PA, United States of America

Chatham Eastside

1 Woodland Road

Pittsburgh, PA 15232

jwasco@chatham.edu 